\title{
Seguimiento farmacoterapéutico: competencia del farmacéutico
}

\author{
Estibaliz Goienetxea Soto \\ Doctora en Farmacia. Directora Técnica. Colegio Oficial de Farmacéuticos de Gipuzkoa.
}

\section{PALABRAS CLAVE}

Farmacia comunitaria, farmacéutico comunitario, competencias, atención farmacéutica, Servicios Profesionales Farmacéuticos

\section{ABREVIATURAS}

ANECA: Agencia Nacional de Evaluación de la Calidad y Acreditación OMS: Organización Mundial de la Salud

\section{KEYWORDS}

Community pharmacy, community pharmacist, competences, pharmaceutical care, Pharmaceutical Services

\section{RESUMEN}

El Seguimiento Farmacoterapéutico (SFT) es un servicio profesional que aborda de manera global los problemas de salud y los medicamentos que utiliza el paciente, centrándose en la valoración de la necesidad, efectividad y seguridad de la farmacoterapia.

Es, por tanto, el farmacéutico el profesional idóneo para llevar a cabo esta actividad, ya que es el experto en el medicamento y tiene el conocimiento, habilidades y actitudes necesarias para abordar todos los campos de actuación. Así queda ampliamente recogido en las competencias profesionales del título de Grado en Farmacia y respaldado por la normativa sanitaria vigente, así como por diversos organismos oficiales nacionales e internacionales, que recomiendan su implantación en farmacia comunitaria.

La implementación de programas sanitarios específicos impulsados por las administraciones públicas, dirigidos a la implantación del servicio de SFT en farmacia comunitaria, supondría una mejora del uso de los medicamentos y la salud de los pacientes, así como un impulso al desarrollo profesional del farmacéutico comunitario y su integración en el Sistema Nacional de Salud (SNS).

\section{Pharmacotherapeutic monitoring pharmacist competency}

\section{ABSTRACT}

The Seguimiento Farmacoterapéutico (SFT), Pharmacotherapeutic Monitoring, is a professional service that approaches globally issues in healthcare and patient medications, focusing on assessing the need for and the safety and efficacy of pharmacological therapy.

Thus, the pharmacist is the suitable professional to carry out this activity, as he/she is the expert in medications and possesses the knowledge, skills and competencies required to approach all fields of action. This is fully reflected in the professional competencies of the title of Degree in Pharmacy and supported by the current healthcare regulation as well as by the various official national and international organizations that recommend its implementation in community pharmacy.

The implementation of specific healthcare programs promoted by public administrations, aimed at implementing the SFT service in community pharmacy, would entail a better use of medications and patient health, as well as an impulse to professional development of community pharmacists and integration in the national Health System (Sistema Nacional de Salud, SNS).
Recibido: 12/10/2017

Aceptado: 15/11/2017

Disponible online: $30-12-2017$
Financiación: ninguna.

Conflicto de intereses: ninguno.

Cite este artículo como: Goienetxea E. Seguimiento farmacoterapéutico: competencia del farmacéutico. Farmacéuticos Comunitarios. 2017 Dec 30; 9(4):14-17. doi:10.5672/FC.2173-9218.(2017/Nol9).004.03 Correspondencia: Estibaliz Goienetxea Soto (egoyenechea@redfarma.org)

ISSN 1885-8619 @SEFAC (Sociedad Española de Farmacia Familiar y Comunitaria). Todos los derechos reservados. 
El Seguimiento Farmacoterapéutico (SFT) se define como el servicio profesional cuyo objetivo es la detección de problemas relacionados con medicamentos (PRM) para la prevención y resolución de resultados negativos asociados a la medicación (RNM) [1]. El SFT aborda de manera global los problemas de salud y los medicamentos que utiliza el paciente, centrándose en la valoración de necesidad, efectividad y seguridad de la farmacoterapia.

Este servicio trata de optimizar la utilización del medicamento para obtener mejoras de salud en el paciente, y es por tanto el farmacéutico el profesional idóneo para llevar a cabo esta actividad, ya que es el experto en el medicamento y tiene el conocimiento, habilidades y actitudes necesarias para abordar todos los campos de actuación.

La adquisición de los conocimientos y habilidades para la realización de SFT quedan claramente recogidas en las competencias profesionales establecidas en el Libro Blanco de la Agencia Nacional de Evaluación de la Calidad y Acreditación (ANECA) del título de Grado en Farmacia [2].

Farmacia (Pág. 194): Competencia B: evaluar los efectos de sustancias con actividad farmacológica. Competencia E: emitir consejo terapéutico y participar en la toma de decisiones en farmacoterapia $y$ dietoterapia, en los ámbitos comunitario, hospitalario, sociosanitario $y$ en atención domiciliaria. Competencia G: identificar, evaluar y valorar los problemas relacionados con los fármacos $y$ medicamentos y participar en las actividades de farmacovigilancia. Competencia H: llevar a cabo las actividades de farmacia clínica $y$ social, siguiendo con el ciclo de atención farmacéutica.

Los Libros Blancos del resto de profesiones sanitarias no recogen competencias relacionadas con el servicio de SFT, por lo que no se adquieren los conocimientos específicos para la realización del servicio. Cada titulado facultativo adquiere las competencias propias del Grado correspondiente, como son el diagnóstico de la enfermedad y establecimiento del tratamiento en el caso del Grado en Medicina, y la promoción de la salud y cuidados del paciente para el Grado en Enfermería. En cuanto a las competencias concretas que los Libros Blancos de estos grados sanitarios establecen en relación a los medicamentos, en el caso de Medicina claramente indica que es de su competencia el establecimiento del tratamiento y la indicación de la terapéutica más adecuada (3], y la administración segura de los medicamentos en el caso del Grado en Enfermería [4]:

Medicina (pág. 89): Competencia 17 en Competencias Clínicas: establecer el diagnostico, pronostico y tratamiento aplicando los principios basados en la mejor información posible. Competencia 18 en Competencias Clínicas: indicar la terapéutica más adecuada de los procesos agudos y crónicos más prevalente, asi como de los enfermos en fase terminal.

Enfermería (pág. 85): Competencia 14 Grupo III: capacidad para administrar con seguridad fármacos y otras terapias (utilizando habilidades...

Por tanto, la realización del servicio de SFT no constituye, en ningún caso, una invasión de competencias por parte del farmacéutico, sino que consiste en poner en práctica aquellas competencias propias que a los graduados en Farmacia les corresponden. Además, conviene remarcar que el servicio de SFT no contempla ni diagnóstico de enfermedad ni prescripción de medicamentos, de ahí que el farmacéutico deba prestar el servicio conjuntamente con el médico y el propio paciente, lo que queda claramente recogido en la definición de Foro de Atención Farmacéutica en Farmacia Comunitaria (Foro AF-FC) [1]:

"El Seguimiento Farmacoterapéutico es el servicio profesional que tiene como objetivo la detección de problemas relacionados con medicamentos (PRM) para la prevención y resolución de resultados negativos asociados a la medicación (RNM). Este servicio implica un compromiso, y debe proveerse de forma continuada, sistematizada y documentada, en colaboración con el propio paciente $y$ con los demás profesionales del sistema de salud, con el fin de alcanzar resultados concretos que mejoren la calidad de vida del paciente".

Este hecho, lejos de verse como un obstáculo, debería entenderse como una oportunidad de colaboración entre distintos profesionales sanitarios con el objetivo común de mejorar la salud de los pacientes. De hecho, el concepto de salud, en su sentido más amplio, implica la plena utilización de todos los recursos sanitarios, potenciando los equipos multidisciplinares de salud (práctica colaborativa) y, en particular, la coordinación médico-farmacéutico en lo relativo al incremento del beneficio terapéutico de los medicamentos.

Además de las competencias profesionales, la normativa sanitaria vigente defiende a través de distintas leyes (enumeradas a continuación), que la realización del servicio de SFT es responsabilidad del profesional farmacéutico.

La Ley de ordenación de las profesiones sanitarias [5], que regula los aspectos básicos de las profesiones sanitarias tituladas y el desarrollo profesional de las mismas, establece entre sus funciones que las actividades dirigidas a la instauración del tratamiento y terapéutica le corresponde a los graduados en Medicina, siendo funciones del graduado en Farmacia la dispensación de los medicamentos y la colaboración en el proceso farmacoterapéutico de los medicamentos. En lo que respecta a las funciones del Grado en Enfermería, destaca la prestación de cuidados a los pacientes, pero no menciona ninguna función relacionada con la terapéutica y el uso de los medicamentos.

Ley 44/2003, de 21 de noviembre, de ordenación de las profesiones sanitarias. A continuación se enumeran las funciones de las profesiones sanitarias de medicina, farmacia y enfermería:

Artículo 6: a) Médicos: corresponde a los licenciados en Medicina la indicación y realización de las actividades dirigidas a la promoción y mantenimiento de la salud, a la prevención de las enfermedades y al diagnóstico, tratamiento, terapéutica y rehabilitación de los pacientes, así como al enjuiciamiento y pronóstico de los procesos objeto de atención. 
b) Farmacéuticos: corresponde a los licenciados en Farmacia las actividades dirigidas a la producción, conservación y dispensación de los medicamentos, así como la colaboración en los procesos analiticos, farmacoterapéuticos y de vigilancia de la salud pública.

Articulo 7: a) Enfermeros: corresponde a los diplomados universitarios en Enfermería la dirección, evaluación y prestación de los cuidados de Enfermería orientados a la promoción, mantenimiento y recuperación de la salud, asi como a la prevención de enfermedades y discapacidades.

La Ley 16/1997, de regulación de servicios de las oficinas de farmacia [6], destaca que uno de los servicios básicos que debe prestar la farmacia comunitaria a la población es el de la información y el seguimiento de los tratamientos farmacológicos a los pacientes.

Artículo 1. Definición y funciones de las oficinas de farmacia.

5. La información y el seguimiento de los tratamientos farmacológicos a los pacientes.

6. La colaboración en el control del uso individualizado de los medicamentos, a fin de detectar las reacciones adversas que puedan producirse y notificarlas a los organismos responsables de la farmacovigilancia.

La Ley 29/2006 de garantías y uso racional de los medicamentos y productos sanitarios [7] establece que el farmacéutico deberá colaborar en el seguimiento farmacoterapéutico de los tratamientos prescritos, a través de los procedimientos de la atención farmacéutica.

Artículo 84.1: Los farmacéuticos, como responsables de la dispensación de medicamentos a los ciudadanos, velarán por el cumplimiento de las pautas establecidas por el médico responsable del paciente en la prescripción, y cooperarán con él en el seguimiento del tratamiento a través de los procedimientos de atención farmacéutica, contribuyendo a asegurar su eficacia y seguridad. Asimismo, participarán en la realización del conjunto de actividades destina- das a la utilización racional de los medicamentos, en particular a través de la dispensación informada al paciente.

Hay que añadir que, distintos organismos oficiales nacionales e internacionales, recomiendan que, para la prevención de los riesgos iatrogénicos de la medicación, debería implantarse el SFT en farmacia comunitaria.

El Documento de Consenso de Atención Farmacéutica del Ministerio de Sanidad [8], cuyo objetivo principal fue establecer las bases de la atención farmacéutica en España y respaldar asimismo la evolución de la profesión farmacéutica, recoge el SFT como uno de los servicios asistenciales del farmacéutico orientado al paciente que consume medicamentos, y lo define como una responsabilidad que corresponde al farmacéutico. Esto queda recogido ampliamente en distintos puntos del documento (pág. 221-241), pero cabe destacar la definición:

Por su importancia entre estas actividades clínicas, se destaca la siguiente función asistencial: SEGUIMIENTO FARMACOTERAPÉUTICO PERSONALIZADO es la práctica profesional en la que el farmacéutico se responsabiliza de las necesidades del paciente relacionadas con los medicamentos mediante la detección, prevención $y$ resolución de problemas relacionados con la medicación (PRM), de forma continuada, sistematizada y documentada, en colaboración con el propio paciente $y$ con los demás profesionales del sistema de salud, con el fin de alcanzar resultados concretos que mejoren la calidad de vida del paciente."

La Organización Mundial de la Salud (OMS) redactó un documento conocido como Informe de Tokio [9], que examinaba las responsabilidades del farmacéutico con respecto a las necesidades del paciente y de la comunidad, fundamento de la Atención Farmacéutica. A lo largo del documento se especifica que el farmacéutico tiene que responsabilizarse de la continuidad de los tratamientos y colaborar con el médico en el seguimiento de los mismos.
Define la atención farmacéutica como un concepto de práctica profesional en el que el paciente es el principal beneficiario de las acciones del farmacéutico. La atención farmacéutica es el compendio de las actitudes, los comportamientos, los compromisos, las inquietudes, los valores éticos, las funciones, los conocimientos, las responsabilidades y las destrezas del farmacéutico en la prestación de la farmacoterapia, con objeto de lograr resultados terapéuticos definidos en la salud y la calidad de vida del paciente.

El Consejo de Europa [10] publicó un acuerdo del Comité de Ministros, donde se clasificaba a la Atención Farmacéutica como elemento esencial en la prevención y reducción de riesgos iatrogénicos de la medicación, la cual debería implantarse de forma sistematizada. Un extracto de este documento indica:

Pharmaceutical care is an essential element in the prevention and reduction of iatrogenic risks and should be implemented systematically. It includes: - the monitoring of prescriptions, particularly in the light of the patient's pharmaceutical record in order to check for consistency and for possible interactions with other medicines; -the evaluation of patients' overall medication.

Además, es un servicio que ha sido consensuado por organizaciones científicas y profesionales, para su implantación en España, como son el Consejo General de Colegios de Farmacéuticos [11,12], y hay que remarcar que su realización en farmacia comunitaria ha quedado respaldada por los resultados obtenidos en distintos estudios de investigación. Un estudio nacional desarrollado recientemente muestra como la realización de SFT en farmacia comunitaria a pacientes mayores polimedicados reduce los problemas de salud, las visitas a urgencias, los ingresos hospitalarios y, además, logra mejorar significativamente la calidad de vida de estos pacientes [13]. Otro estudio concluye que la realización de SFT en farmacia comunitaria reduce el número de medicamentos inapropiados y aumenta la adherencia de los pacientes mayores [14]. Además, la implantación de 
SFT en farmacia comunitaria se ha descrito como un servicio coste-efectivo [15], que puede suponer unos importantes ahorros para el Sistema Nacional de Salud (SNS) [16,17], de ahí que la prestación del servicio de SFT a pacientes mayores polimedicados se haya convertido en el centro de atención de las políticas del Gobierno central y de las comunidades autónomas. Pese a esto, el proceso de su implantación está siendo lento y requiere un esfuerzo considerable por parte de los farmacéuticos, así como un cambio en los aspectos organizativos de la farmacia. Para que esto ocurra faltan los condicionantes necesarios para su implantación, como son la necesidad de implementar programas sanitarios específicos impulsados por las administraciones públicas y su inclusión como un servicio eficiente y necesario en la cartera de servicios del SNS, que además genera rentabilidad a su proveedor.

Con la implantación del servicio de SFT en farmacia comunitaria, se lograría disminuir la morbi-mortalidad relacionada con los medicamentos, cubrir una necesidad sanitaria, desarrollar las competencias profesionales de los farmacéuticos y su integración en el SNS, fomentar la labor multidisciplinar de los profesionales sanitarios, generar ahorros al sistema, incluso cuando se remunera por la realización del servicio y, en definitiva y lo más importante, mejorar el uso de los medicamentos y la salud de los pacientes.

\section{Referencias bibliográficas}

1. Foro AF-FC [Internet]. Guía Práctica para los Servicios de Atención Farmacéutica en la farmacia Comunitaria. Consejo General de Colegios Oficiales de Farmacéuticos. 2010 [Consultado
24/6/2017]. Disponible en: http:// www.portalfarma.com/Inicio/atencionfarmaceutica/forofarmaciacomunitaria/Documents/ATFC_Guia\%20 FORO.pdf

2. Libro blanco del Título de Grado en Farmacia - ANECA [Internet]. [Acceso 22/6/2017]. Disponible en: http://http://www.aneca.es/var/media/150368/libroblanco_farmacia_ def.pdf

3. Libro blanco del Título de Grado en Medicina - ANECA [Internet]. [Acceso 22/6/2017]. Disponible en: http:// www.aneca.es/var/media/150312/libroblanco_medicina_def.pdf

4. Libro blanco del Título de Grado en Enfermeria - ANECA [Internet]. [Acceso 22/6/2017]. Disponible en: http:// www.aneca.es/var/media/150360/libroblanco_jun05_enfermeria.pdf

5. Ley $44 / 2003$, de 21 de noviembre, de ordenación de las profesiones sanitarias (BOE n 280 - 22/11/2003)

6. Ley 16/1997, de 25 de abril, de Regulación de Servicios de las Oficinas de Farmacia (BOE n 100 - 26/4/1997)

7. Ley $29 / 2006$, de 26 de julio, de garantías y uso racional de los medicamentos y productos sanitarios (BOE $n^{\circ} 100$ $-27 / 7 / 2006)$

8. Panel de Consenso. Consenso sobre Atención Farmacéutica. Madrid: Ministerio de Sanidad y Consumo; 2001.

9. Organización Mundial de la Salud. Informe de la Reunión de Tokio (1993) sobre el papel del farmacéutico en el sistema de atención de salud. Pharm Care Esp. 1999; 1(3):207-211.

10. Consejo de Europa. Comité de Ministros. Resolución ResAP (2001) 2 relativa al papel del farmacéutico en el marco de la seguridad de la salud. Pharm Care Esp. 2001; 3(3): 216-222.

11. Grupo de Expertos de Foro de Atención Farmacéutica. Documento de Consenso. Madrid: Consejo General de Colegios Oficiales de Farmacéuticos; 2008.

12. Consejo General de Colegios Oficiales de Farmacéuticos [Internet]. Buenas prácticas en Farmacia Comunitaria en España. Servicio de Seguimiento
Farmacoterapéutico en farmacia comunitaria. 2014. [Acceso 22/6/2017]. Disponible en: http://www.portalfarma.com/Profesionales/Buenas-practicas-profesionales/Documents/BBPP-SERVICIO-SFT-DEFINITIVO.pdf

13. Programa conSIGUE [Internet]. Medida del impacto clínico, económico y humanístico del servicio de Seguimiento Farmacoterapéutico en mayores polimedicados en la farmacia comunitaria española. Informe 20112014. [Acceso 22/6/2017]. Disponible en: http://www.portalfarma.com/ Profesionales/Investigacion Farmacia/conSIGUE/Documents/Resultados-Definitivos-Programa-Consigue-Impacto-2011-2014.pdf

14. Sáez-Benito L, Fernandez-Llimós F, Feletto E, Gastelurrutia MA, Martinez-Martinez F, Benrimoj SI. Evidence of the clinical effectiveness of cognitive pharmaceutical services for aged patients. Age Ageing. 2013 Jul; 42(4):442-9. doi:10.1093/ageing/ aft045

15. Jódar-Sánchez F, Malet-Larrea A, Martín JJ, García-Mochón L, López del Amo MP, Martínez-Martínez F, et al. Cost-utility analysis of a medication review with follow-up service for older adults with polypharmacy in community pharmacies in Spain: the conSIGUE program. Pharmacoeconomics. 2015 Jun; 33(6):599-610. doi:10.1007/s40273-015-0270-2

16. Malet-Larrea A, Goyenechea E, Gastelurrutia MA, Calvo B, García-Cárdenas V, Cabases JM, et al. Cost analysis and cost-benefit analysis of a medication review with follow-up service in aged polypharmacy patients. Eur J Health Econ. 2016 Dec; 18(9):10691078. doi:10.1007/s 10198-016-0853-7

17. Noain A, Garcia-Cardenas V, Gastelurrutia MA, Malet-Larrea A, Martinez-Martinez F, Sabater-Hernandez D, et al. Cost analysis for the implementation of a medication review with follow-up service in Spain. Int J Clin Pharm. 2017 Aug; 39(4):750-758. doi:10.1007/s11096017-0454-2 